\title{
On closed rotating worlds
}

\author{
Thoralf Chrobok, Yuri N. Obukhov*, and Mike Scherfner ${ }^{\dagger}$ \\ Institute of Theoretical Physics, Technical University of Berlin, Hardenbergstr. 36, D-10623 \\ Berlin, Germany
}

\begin{abstract}
A new solution for the stationary closed world with rigid rotation is obtained for the spinning fluid source. It is found that the spin and the vorticity are locally balanced. This model qualitatively shows that the local rotation of the cosmological matter can be indeed related to the global cosmic vorticity, provided the total angular momentum of the closed world is vanishing.
\end{abstract}

Typeset using REVTEX

*On leave from: Department of Theoretical Physics, Moscow State University, 117234 Moscow, Russia

${ }^{\dagger}$ FB Mathematik, Technical University of Berlin, Str. d. 17. Juni 136, D-10623 Berlin 


\section{INTRODUCTION}

The study of rotating cosmological models is of interest both mathematically and physically (some of the previous work on this subject is overviewed in [四,2]). The first stationary metric with vorticity [3, 1] described an open world filled with dust matter plus the cosmological term. For many years and for the numerous researchers, this model served a kind of theoretical laboratory for the investigation of the specific rotational effects. Besides, it stimulated the study of the role of Mach's principle in the general relativity theory. In this respect, it is worthwhile to mention the papers of Ozsváth and Schücking [5] which made a major contribution by providing the true anti-Machian model. The latter describes a spatially closed world, in contrast to the open universe of Gödel.

One can distinguish the two types of rotation in cosmology. The first one, which can be called geometrical (or spacetime) rotation, is determined by the kinematics of the curved spacetime itself, irrespectively of the matter contents of a model. Technically, its properties are described by a formal comoving observer interpreting the cosmic time coordinate line as an own world-line. The second type of rotation arises when the cosmological matter itself has a proper motion such that the corresponding fluid flow is characterized by the nontrivial vorticity (which can be called material rotation).

In the original Gödel's model [3], these two rotations coincide because the matter is assumed comoving, however this is not true in general [6]. A typical example is provided in [7] where the matter flow has nontrivial vorticity, whereas the spacetime (described by the Robertson-Walker metric) is non-rotating. Another example is given by the anti-Machian closed world model [5] in which the material and geometrical rotations are different (moreover, the matter flow has a nontrivial shear unlike the spacetime geometry itself).

In the study of the rotating cosmological models, it seems natural to consider the spin (intrinsic angular momentum) as an important physical property of the matter source. After all, the elements of the "cosmic fluid" are either galaxies or clusters of galaxies (at modern epoch) or the high energy elementary particles (at early epochs) all of which have spin. 
Actually, that was one of the basic motivations for Whittaker [0] to ask how can the largest physical system (universe) be non-rotating when all the other known systems, from particles to galaxies, have rotation.

In the present paper, we will demonstrate that the spinning cosmic matter gives rise to a true anti-Machian rotating closed world. The resulting model is, in a certain sense, a "relative" of the old stationary model of Ozsváth and Schücking [5]. Namely, both models belong to the type-IX Bianchi spatially homogeneous spacetimes. There is however, an essential difference in that the material rotation now coincides with the spacetime rotation (i.e., there is no proper motion of matter) and the shear is absent, in contrast to [5]. It seems worthwhile to note that our new model is stationary and thus, like the Ozsváth and Schücking world [5], it does not refer directly to the real expanding universe.

\section{SPINNING MATTER}

We will describe the cosmic matter with spin by means of the Weyssenhoff model. It was proposed in flat spacetime in [9], and the modern treatment of the curved spacetime variational theory of spinning fluid is presented in [10].

The ideal Weyssenhoff fluid is a continuous medium, the elements of which are characterized by the intrinsic angular momentum (spin) proportional to the volume element. The spin density is described by the second rank skew-symmetric tensor

$$
S^{i j}=-S^{j i},
$$

the spatial components of which coincide in the rest frame with the density of the 3 -vector of spin of a matter element. This is provided by imposing the covariant constraint, the so called Frenkel condition:

$$
S_{i j} u^{i}=0,
$$

where $u^{i}$ is the four-velocity of a fluid element. 
The (metric) energy-momentum tensor of the Weyssenhoff fluid reads:

$$
T_{i j}=\varepsilon u_{i} u_{j}-p h_{i j}-2\left(g^{k l}+u^{k} u^{l}\right) \nabla_{k}\left(u_{(i} S_{j) l}\right),
$$

where $\varepsilon$ is the energy density, $p$ is the pressure, and $h_{i j}=g_{i j}-u_{i} u_{j}$ is the standard projector (with the velocity normalized by $u_{i} u^{i}=1$ ). The energy-momentum tensor (3) can be written in a different form of a general non-ideal fluid with "the energy flux" $q^{i}$ and "anisotropic pressure" $\pi_{i j}$ as

$$
T_{i j}=\varepsilon_{\mathrm{eff}} u_{i} u_{j}-p_{\mathrm{eff}} h_{i j}+2 u_{(i} q_{j)}+\pi_{i j},
$$

where as usually $u^{i} q_{i}=0, \pi_{i}^{i}=0, \pi_{i j} u^{i}=0$, and the newly introduced quantities are defined by the spin:

$$
\begin{aligned}
\varepsilon_{\mathrm{eff}} & =\varepsilon-2 \omega_{k l} S^{k l}, \quad p_{\mathrm{eff}}=p-\frac{2}{3} \omega_{k l} S^{k l}, \\
q_{i} & =-\left(h^{j}{ }_{i} \nabla_{l} S_{j}{ }^{l}+a^{l} S_{l i}\right), \\
\pi_{i j} & =2 \omega_{(i}{ }^{k} S_{j) k}-2 \sigma_{(i}{ }^{k} S_{j) k}-\frac{2}{3} h_{i j} \omega_{k l} S^{k l} .
\end{aligned}
$$

The dynamics of spinning fluid is described by the rotational and translational equations of motion. The rotational equations give the motion of spin:

$$
\nabla_{i}\left(u^{i} S_{k l}\right)=u_{k} u^{j} \nabla_{i}\left(u^{i} S_{j l}\right)-u_{l} u^{j} \nabla_{i}\left(u^{i} S_{j k}\right)=u_{k} S_{l j} a^{j}-u_{l} S_{k j} a^{j}
$$

Hereafter $a^{j}=u^{i} \nabla_{i} u^{j}$ is the acceleration field. The translational equations of motion are, as usual, the consequence of the energy-momentum conservation law. From (3) one finds, with the help of (2), (8) and the Ricci identity:

$$
\begin{aligned}
\nabla_{i} T_{j}^{i}= & u_{j}\left(u^{i} \nabla_{i} \varepsilon+\varepsilon \nabla_{i} u^{i}+p \nabla_{i} u^{i}\right)-h_{j}^{i} \nabla_{i} p \\
& +(p+\varepsilon) a_{j}+2 u^{i} S_{j l} \nabla_{i} a^{l}+R_{k l i j} u^{i} S^{k l}=0
\end{aligned}
$$

Projections of (9) on $u^{i}$ and on the orthogonal directions read

$$
\begin{aligned}
(p+\varepsilon) \nabla_{i} u^{i}+u^{i} \nabla_{i} \varepsilon & =0, \\
(p+\varepsilon) a_{j}-h_{j}^{i} \nabla_{i} p+2 S_{j i} u^{l} \nabla_{l} a^{i}+R_{k l i j} u^{i} S^{k l} & =0 .
\end{aligned}
$$




\section{GEOMETRY}

We will study the stationary spacetime model belonging to the wide class of rotating spatially homogeneous models [2]

$$
d s^{2}=d t^{2}-2 R n_{a} d x^{a} d t-R^{2} \gamma_{a b} d x^{a} d x^{b}
$$

Hereafter the indices $a, b, c=1,2,3$ label the spatial coordinates, $R=R(t)$ is the scale factor, and

$$
n_{a}=\nu_{A} e_{a}^{A}, \quad \gamma_{a b}=\beta_{A B} e_{a}^{A} e_{b}^{B}
$$

Here $\nu_{A}, \beta_{A B}$ are constant coefficients $(A, B=1,2,3)$, while

$$
e^{A}=e_{a}^{A}(x) d x^{a}
$$

are the invariant 1 -forms with respect to the action of a three-parameter group of motion

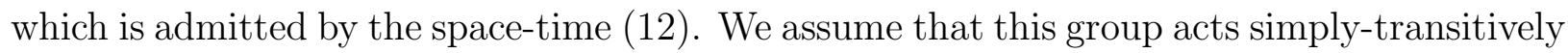
on the spatial ( $t=$ const) hypersurfaces. It is well known that there exist 9 types of such manifolds, distinguished by the Killing vectors $\xi_{A}$ and their commutators $\left[\xi_{A}, \xi_{B}\right]=f_{A B}^{C} \xi_{C}$.

Invariant forms (14) solve the Lie equations $\mathcal{L}_{\xi_{B}} e^{A}=0$ for each Bianchi type, so that models (12) are spatially homogeneous.

More specifically, we will confine our attention to the case of the closed world belonging to the Bianchi type IX. Then, denoting the spatial coordinates $x=x^{1}, y=x^{2}, z=x^{3}$, one has explicitly:

$$
e^{1}=\cos y \cos z d x-\sin z d y, \quad e^{2}=\cos y \sin z d x+\cos z d y, \quad e^{3}=-\sin y d x+d z,
$$

which satisfy the structure equations

$$
d e^{A}=f^{A}{ }_{B C} e^{B} \wedge e^{C}, \quad \text { with } \quad f^{1}{ }_{23}=f^{2}{ }_{31}=f^{3}{ }_{12}=1 .
$$

We now write the ansatz for the line element (12) 


$$
d s^{2}=g_{\alpha \beta} \vartheta^{\alpha} \vartheta^{\beta}, \quad g_{\alpha \beta}=\operatorname{diag}(1,-1,-1,-1),
$$

in terms of the orthonormal coframe 1-forms $\vartheta^{\alpha}$ :

$$
\vartheta^{\widehat{0}}=d t-R \nu_{A} e^{A}, \vartheta^{\widehat{1}}=R k_{1} e^{1}, \vartheta^{\widehat{2}}=R k_{2} e^{2}, \vartheta^{\widehat{3}}=R k_{3} e^{3} .
$$

Here, $k_{1}, k_{2}, k_{3}$ are positive constant parameters. The Greek indices $\alpha, \beta, \ldots=0,1,2,3$ hereafter label the objects with respect to the orthonormal frame; the hats over indices denote the separate frame components of these objects.

\section{CLOSED ROTATING WORLD}

In order to finalize the formulation of the problem, we have to specify the assumptions about the cosmic fluid source. As we already mentioned, we are interested in the comoving case, when the mean velocity is

$$
u^{i}=\delta_{0}^{i}
$$

Hence, with respect to the orthonormal frame, $u^{\alpha}=(1,0,0,0)$, and from the Frenkel condition (21) we see that the spin density can have only spatial nontrivial components:

$$
S_{\widehat{2} \widehat{3}}, \quad S_{\widehat{31}}, \quad S_{\widehat{12}} .
$$

We are looking for the stationary world, so all the time dependence is absent, and thus the scale factor is fixed at some constant value, $R=R_{0}$, whereas the components of spin (20) are also constant.

The gravitational field equations read, with $\kappa$ the Einstein gravitational constant and cosmological constant $\Lambda$ (with respect to the orthonormal local frame):

$$
R_{\alpha \beta}-\frac{1}{2} R g_{\alpha \beta}=\kappa T_{\alpha \beta}+\Lambda g_{\alpha \beta} .
$$

Substituting (18)-(20) into (3) and (21), after some algebra one finds from the off-diagonal components of the Einstein equations: 


$$
\kappa S_{\widehat{2} \widehat{3}}=\frac{\nu_{1}}{2 R_{0} k_{2} k_{3}}, \quad \kappa S_{\widehat{3} \widehat{1}}=\frac{\nu_{2}}{2 R_{0} k_{1} k_{3}}, \quad \kappa S_{\widehat{12}}=\frac{\nu_{3}}{2 R_{0} k_{1} k_{2}} .
$$

Using these in (8), one verifies that the rotational equations of motion are satisfied automatically. Substituting (22) into the diagonal components of (21), one finds that the latter are consistent only when all the three parameters are equal:

$$
k_{1}=k_{2}=k_{3}=: k
$$

Then, the Einstein equations yield for the energy density and the pressure

$$
\begin{aligned}
\kappa \varepsilon+\Lambda & =\frac{3 k^{2}-\nu_{1}^{2}-\nu_{2}^{2}-\nu_{3}^{2}}{4 R_{0}^{2} k^{4}}, \\
\kappa p-\Lambda & =\frac{-k^{2}-\nu_{1}^{2}-\nu_{2}^{2}-\nu_{3}^{2}}{4 R_{0}^{2} k^{4}} .
\end{aligned}
$$

For the dust equation of state $p=0$ (incoherent matter), we thus finally find:

$$
\varepsilon=\frac{2\left(k^{2}-\nu_{1}^{2}-\nu_{2}^{2}-\nu_{3}^{2}\right)}{4 \kappa R_{0}^{2} k^{4}}, \quad \Lambda=\frac{k^{2}+\nu_{1}^{2}+\nu_{2}^{2}+\nu_{3}^{2}}{4 \kappa R_{0}^{2} k^{4}} .
$$

\section{DISCUSSION AND CONCLUSION}

It is straightforward to obtain the kinematical quantities which describe the spacetime geometry. A direct calculation of the vorticity, $\omega_{\mu \nu}=h^{\alpha}{ }_{\mu} h^{\beta}{ }_{\nu} \nabla_{[\alpha} u_{\beta]}$, shear, $\sigma_{\mu \nu}=$ $h^{\alpha}{ }_{\mu} h^{\beta}{ }_{\nu} \nabla_{(\alpha} u_{\beta)}-\frac{1}{3} h_{\mu \nu} \nabla_{\lambda} u^{\lambda}$, and the volume expansion $\theta=\nabla_{\lambda} u^{\lambda}$, yields:

$$
\begin{aligned}
& \sigma_{\mu \nu}=0, \quad a^{\alpha}=0, \quad \theta=0, \\
& \omega_{\widehat{23}}=-\frac{\nu_{1}}{2 R_{0} k^{2}}, \quad \omega_{\widehat{3} \widehat{1}}=-\frac{\nu_{2}}{2 R_{0} k^{2}}, \quad \omega_{\widehat{1} \widehat{2}}=-\frac{\nu_{3}}{2 R_{0} k^{2}} .
\end{aligned}
$$

Comparing with (22), we find

$$
\kappa S_{\alpha \beta}=-\omega_{\alpha \beta}
$$

This is quite a remarkable relation as it indeed supports the idea which was put forward by Whittaker [8]: the rotation of the local matter elements, i.e. spin $S_{\alpha \beta}$, is derived from 
the overall global rotation of the universe, that is $\omega_{\alpha \beta}$. Moreover this result is in complete agreement with the work of King [11] on closed rotating Bianchi-IX spacetimes. The rotation of the local matter elements are balanced by the rotation of the spacetime, so that there is no net angular momentum. This can be made more explicit by recasting (29) into the form of the equation describing the vanishing of the total (orbital plus spin) angular momentum of the universe,

$$
\kappa S_{\alpha \beta}+\omega_{\alpha \beta}=0
$$

Thus, in the sense of King, our solution is not a counterexample to Mach's principle. We however have a more direct and transparent mechanism of the balance, without the need of the gravitational-wave interpretation developed in [11]. Note that our results have been obtained in the framework of Einstein's general relativity theory. Similar observations were also reported for the cosmic spinning string solutions in the Einstein-Cartan theory of gravity [12].

Another interesting result is that, given the radius of the universe $R_{0}$, the magnitude of the angular velocity $\omega^{2}:=\frac{1}{2} \omega_{\alpha \beta} \omega^{\alpha \beta}=\left(\nu_{1}^{2}+\nu_{2}^{2}+\nu_{3}^{2}\right) /\left(4 R_{0}^{2} k^{4}\right)$ cannot be arbitrarily large in the closed world. As we see from (26), the energy density is only positive when

$$
|\omega|<\frac{1}{2 R_{0} k}
$$

Recall that in the open Gödel $\operatorname{cosmos} \kappa \varepsilon=2 \omega^{2}$, and thus the angular velocity could be arbitrary.

\section{ACKNOWLEDGMENTS}

This work was supported by the Deutsche Forschungsgemeinschaft with the grants HE 1922/5-1 and 436 RUS 17/72/00. 


\section{REFERENCES}

[1] M. Scherfner, T. Chrobok, and M. Shefaat, Eds., Colloquium on Cosmic Rotation (Wissenschaft und Technik Verlag: Berlin, 2000).

[2] Yu.N. Obukhov, in: "Colloquium on Cosmic Rotation" (Berlin, Feb. 1998), Eds. M. Scherfner, T. Chrobok, and M. Shefaat (Wissenschaft und Technik Verlag: Berlin, 2000) 23-96; E-print archive: astro-ph/0008106.

[3] K. Gödel, Rev. Mod. Phys. 21 (1949) 447.

[4] K. Gödel, in: Proc. of Int. Congress of Mathematicians (Cambridge, USA, 1950), Eds. L.M. Graves, E. Hille, P.A. Smith, and O. Zariski, (American Math. Soc.: Providence, RI, 1952) v. 1, 175.

[5] I. Ozsváth and E. Schücking, Nature 193 (1962) 1168; I. Ozsváth and E. Schücking, in: Recent developments in general relativity (Pergamon: Oxford, 1962) 339; I. Ozsváth and E. Schücking, Ann. Phys. 55 (1969) 166. I. Ozsváth and E.L. Schücking, J. Geom. Phys. 24 (1998) 303; I. Ozsváth, Class. Quantum Grav. 14 (1997) A291.

[6] V.F. Panov, Sov. Phys. J. 28 (1985) 18; V.F. Panov, Sov. Phys. J. 28 (1985) 981; V.F. Panov, Sov. Phys. J. 30 (1987) 772.

[7] Ø. Grøn and H.H. Soleng, J. Math. Phys. 29 (1988) 1514; Erratum, J. Math. Phys. 29 (1988) 2320 .

[8] E.T. Whittaker, Spin in the universe, Yearbook of Roy. Soc. Edinburgh (1945) 5.

[9] J. Weyssenhoff and A. Raabe, Acta Phys. Pol. 9 (1947) 7.

[10] Yu.N. Obukhov and V.A. Korotky, Class. Quantum Grav. 4 (1987) 1633; Yu.N. Obukhov and O.B. Piskareva, Class. Quantum Grav. 6 (1989) L15.

[11] D.H. King, A closed universe cannot rotate, in: Einstein Studies, vol. 6: "Mach's principle: From Newton's bucket to quantum gravity", Eds. J.B. Barbour and H. Pfister 
(Birkhäuser: Boston, 1995) 237; D.H. King, Phys. Rev. D44 (1991) 2356.

[12] H.H. Soleng, Gen. Rel. Grav. 24 (1992) 111. 